\title{
Total knee arthroplasty status and patient-reported, knee-related quality of life over a 4-year follow-up period: data from the osteoarthritis initiative
}

This article was published in the following Dove Press journal: Patient Preference and Adherence

\author{
Saad M Bindawas \\ Department of Rehabilitation \\ Sciences, College of Applied Medical \\ Sciences, King Saud University, \\ Riyadh, Saudi Arabia
}

Purpose: To examine the relationship between baseline total knee arthroplasty (TKA) status and patient-reported, knee-related quality of life $(\mathrm{QoL})$ over a 4-year follow-up relative to no knee osteoarthritis (OA).

Patients and methods: Data from the Osteoarthritis Initiative were included, comprising 4,674 adults aged 45-79 years at baseline. Patients were categorized into four groups: no knee OA $(n=3,711)$, non-TKA $(n=902)$, pre-TKA $(n=36)$, and post-TKA $(n=25)$. QoL was measured using the Knee Injury Osteoarthritis Outcome Score-QoL subscale. General linear mixed models were used for the relationship between TKA and QoL.

Results: After adjusting for covariates, a reduced QoL was noted for patients in the non-TKA (mean [standard error], -13.97 [0.73]; $P<0.0001)$, pre-TKA ( -21.34 [3.57]; $P<0.0001)$, and post-TKA ( -9.68 [3.94]; $P=0.0143)$ groups compared with the no knee OA group. QoL in the non-TKA group also decreased over time $(-0.16$ [0.07]; $P=0.226)$.

Conclusion: Non-, pre-, and post-TKA status is associated with a lower QoL.

Keywords: arthroplasty, osteoarthritis, quality of life, knee

\section{Introduction}

Total knee arthroplasty (TKA) is a surgical procedure, with excellent survival rates of 15-20 years routinely reported. ${ }^{1}$ TKA has revolutionized the quality of life (QoL) of millions of patients and has proven to be a cost-effective and reliable treatment for knee osteoarthritis (OA). ${ }^{2,3}$ Although most patients treated with TKA report decreased pain and improved function, nearly one in five patients report a reduced QoL, ${ }^{4,5}$ the reasons for which are not well documented in the literature. ${ }^{5,6}$

Knee-related QoL after TKA is determined using patient-reported outcomes and clinician assessments. ${ }^{7,8}$ Patient-reported outcomes are frequently used in clinical research ${ }^{9}$ and routinely in health care $^{10}$ for evaluating QoL following TKA. ${ }^{11}$ Among the few disease-specific, patient-reported outcome instruments, the Knee Injury and Osteoarthritis Outcome Score-QoL (KOOS-QoL) subscale measures QoL in patients with knee OA, ${ }^{12,13}$ evaluating knee-specific mental and social aspects of knee symptoms that impact on patient QoL. ${ }^{14}$ The KOOS-QoL subscale is regarded as the most reliable and valid patient-reported outcome instrument that considers QoL after TKA. ${ }^{14,15}$ A recently published prospective cohort study evaluated the change in QoL after TKA according to patient-reported outcomes, ${ }^{8}$ and found that patients generally reported improved QoL at 1 year after TKA.
Correspondence: Saad M Bindawas Department of Rehabilitation Sciences, College of Applied Medical Sciences, King Saud University, PO Box 10219 , Riyadh II 433, Saudi Arabia

Tel +966 I| 4696226

Fax+966 II 4693589

Email sbindawas@ksu.edu.sa 
No previous studies have examined the patient-reported QoL over a 4-year follow-up in patients grouped according to their TKA and OA status. Therefore, the purpose of this study was to examine the relationship between baseline TKA status and patient-reported QoL over a 4-year follow-up period and to compare QoL with patients with no knee OA. It was hypothesized that treatment with TKA would reduce the patient-reported decline in QoL in patients with OA over a 4-year follow-up period.

\section{Patients and methods}

In this 4-year longitudinal study, data were obtained from the Osteoarthritis Initiative (OAI). The specific datasets used correspond to version numbers: $0.2 .2,1.2 .1$, 3.2.1, 5.2.1, and 6.2.1. The OAI is a publicly and privately funded, multicenter, ongoing prospective cohort study. The OAI recruited and enrolled men and women $(\mathrm{N}=4,796)$ across four clinical centers (Baltimore, MA; Columbus, OH; Pittsburgh, PA; and Pawtucket, RI) in the United States from February 2004 to May 2006. A detailed description of the OAI objectives and protocol are available freely. ${ }^{26}$ OAI recruited two primary subcohorts: 1) progression subcohort, consisting of 1,390 participants with symptomatic tibiofemoral knee OA in at least one primary knee at baseline; 2) incidence subcohort, consisting of 3,284 participants with an elevated risk of developing symptomatic knee OA during the study.

Of the 4,796 participants, 4,674 participants were included aged between 45 and 79 years at baseline. Participants $(n=122)$ who had no pain, aching, or stiffness; no radiographic findings of $\mathrm{OA}$, and no eligibility risk factors in either knee in the past year were excluded from the analyses. All 4,674 participants at baseline were categorized into the following four groups, according to TKA or OA status, as confirmed by baseline X-ray: no knee OA ( $\mathrm{n}=3,711)$, non-TKA $(\mathrm{n}=902)$, pre-TKA $(n=36)$, and post-TKA $(n=25)$. There were no dropouts over the 4-year study period. Participants in the "no OA knee" group were those who, at baseline, had no symptomatic tibiofemoral knee OA in either knee. Participants in the non-TKA group were those who showed symptomatic tibiofemoral knee OA in either knee who were not considering (and did not receive) surgical intervention but were treated conservatively over the 4 years. Participants categorized as pre-TKA were those with $\mathrm{OA}$ in either knee considering TKA in the next 3 years, whereas those categorized as postTKA were participants who had been treated with TKA in either knee.
The KOOS was developed to evaluate patients' opinions about their knees and associated problems, ${ }^{14}$ and it comprises 42 items across 5 subscales: 1) pain, 2) other symptoms, 3) activities of daily living, 4) function in sport and recreation, and 5) knee-related QoL. ${ }^{16}$ The KOOS-QoL subscale is used to assess patient-reported $\mathrm{QoL}^{17}$ and comprises 4 items: 1) how often is the patient aware of their knee problem, 2) does the patient avoid potentially damaging activities, 3) how much is the patient troubled by the lack of confidence in their knee, and 4) how much difficulty does the patient have with their knee. ${ }^{14}$ Scores are given for every item in the subscale using a Likert scale, ranging from 0 (no problem) to 4 (extreme problems). A QoL score is then calculated by summing the item scores and the data transformed to a $0-100$ scale, with zero representing extreme knee problems (low QoL) and 100 representing no knee problem (high QoL).

\section{Statistical analysis}

The descriptive statistics are summarized using the mean and standard deviation (SD) for continuous variables and count (percentage) for categorical variables. Pearson's $\chi^{2}$, analysis of variance, or post hoc Tukey tests were used to examine the distribution of covariates for participants by their TKA/OA status at baseline. General linear mixed models using the MIXED procedure were used to examine the patient-reported QoL (KOOS-QoL) over the 4-year period according to the participants TKA/OA status at baseline. We used autoregressive covariance matrices; this method uses all possible data for each subject and supports the use of realistic variance and correlation patterns. ${ }^{18}$

Two mixed models were constructed to examine the relationship between TKA/OA status and change in QoL over the 4-year period. Model 1 included group, time, age, sex, race, marital status, education, annual income, and health care coverage. Model 2 included all of the variables in Model 1 plus depressive symptoms, Physical Activity Scale for Elderly (PASE), body mass index (BMI), and comorbidities. Both models used an interaction term between non-, pre-, and post-TKA and time.

The two mixed models provided estimates of the leastsquares means (standard error [SE]) at each time point (baseline, 1-, 2-, 3-, and 4-years). Model 1 included the interactions with time, age, sex, race, marital status, education, annual income, and health care coverage. Model 2 incorporated all of the variables in Model 1 plus interaction with time, depressive symptoms, PASE, BMI, and comorbidities. The primary purpose of including a covariate in the analysis was to ascertain a high or modest relationship between the covariate and the outcome 
measure, as suggested in the literature..$^{19}$ All examinations were performed using the statistical analysis software (SAS) ${ }^{20}$ for Windows, version 9.2 (SAS Institute, Inc., Cary, NC, USA).

\section{Results}

Of the 4,796 participants at baseline, 4,674 participants were included in the current study. Among these 4,674 participants, 3,711 participants had no knee OA (79\%), 902 had OA treated as non-TKA (19\%), 36 were treated as pre-TKA $(1.5 \%)$, and 25 had been treated with TKA and were treated as post-TKA $(0.5 \%)$. The number of participants in each group was the same over the 4-year period (Figure 1).

The mean age of the 4,676 participants was $63.0 \pm 8.8$ years at baseline. Most were female (54\%), white, or Caucasian (75\%), had more than a high school-level education (79\%), and had health care coverage (93\%). The mean BMI score was 30.0 \pm 5.0 , the mean PASE score was $144.0 \pm 81.4$, and the mean KOOS-QoL score was 57.0 \pm 24.3 . Table 1 presents the baseline descriptive characteristics of the sample by TKA/ OA status. The mean KOOS-QoL scores were 69.7 \pm 28.3 for participants in the no knee OA group, $52.3 \pm 19.7$ for the non-TKA group, 39.0 \pm 22.9 for the pre-TKA group, and 66.0 \pm 26.6 for the post-TKA group. Participants in the post-TKA group were significantly older than the other 3 groups (by 9 years) and had a lower average PASE score (121.1 \pm 70.4$)$. Participants in the pre-TKA group were

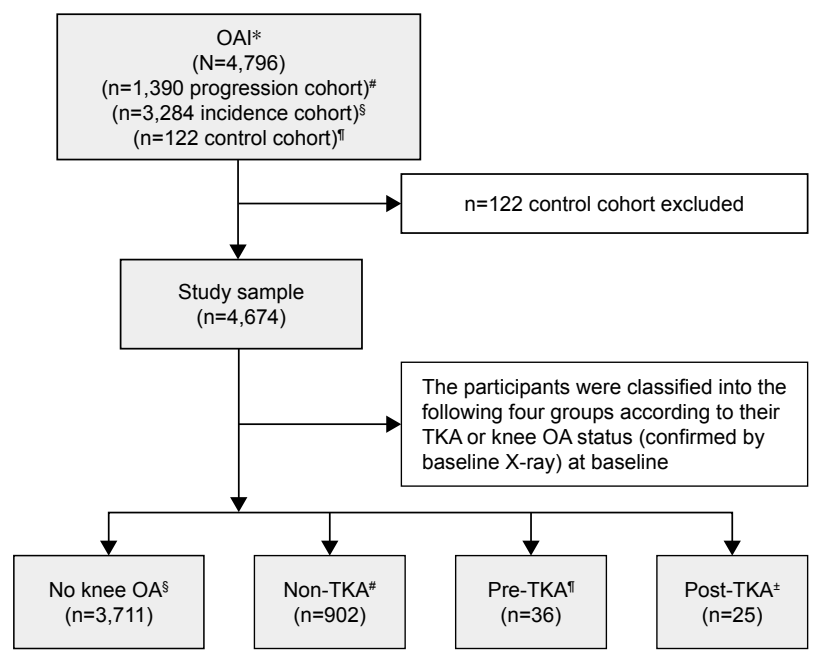

Figure I Flow of the study sample.

Notes: *The Osteoarthritis Initiative Study has made large, heterogeneous datasets available for public use. ${ }^{26}$ "Participants with symptomatic tibiofemoral knee OA at baseline. Participants with no symptomatic tibiofemoral $O A$ in either knee at

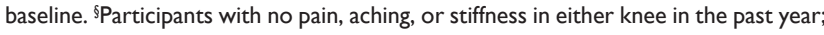
no radiographic findings of $\mathrm{OA}$; and no eligibility risk factors. "Participants considering, for either knee, knee replacement surgery in the next 3 years. ${ }^{ \pm}$Participants, for either knee, had undergone TKA at baseline, confirmed by baseline $\mathrm{X}$-ray. Abbreviations: TKA, total knee arthroplasty; OA, osteoarthritis; OAl, Osteoarthritis Initiative. significantly younger, had less annual income (41\%), high comorbidities, and high mean BMI (31.5 \pm 5.9$)$. Participants in the no knee OA group and non-TKA group had the same

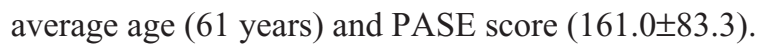

Table 2 shows the general linear mixed models estimates for the KOOS-QoL subscale scores over the 4-year period by TKA/OA status at baseline. The adjusted rate of decline for the KOOS-QoL subscale score was 0.38 per year. After adjusting for age, sex, race, marital status, education, annual income, health care coverage, and time (Model 1), a significant decrease in the KOOS-QoL subscale scores for the non-TKA group (mean [SE], -14.28 [0.74]; $P<0.0001$ ), the pre-TKA group (-21.50 [3.41]; $P<0.0001)$, and post-TKA group $(-9.42$ [3.91]; $P=0.0161)$ were found as compared with the no knee OA group. Considering the interaction term between non-, pre-, and post-TKA and time of follow-up (slope of KOOS-QoL subscale score over 4 years), a significant decline in the KOOS-QoL subscale scores for the non-TKA group $(-0.15$ [0.07]; $P=0.0302)$ was found.

In Model 2, after controlling for depressive symptoms, PASE, BMI, and comorbidities, together with the variables in Model 1, the non-TKA group $(-13.97$ [0.73]; $P<0.0001)$, pre-TKA group $($ Estimate $=-21.34$ [3.57]; $P<0.0001)$, and post-TKA group ( -9.68 [3.94]; $P=0.0143$ ) all remained significantly associated with a decreased KOOS-QoL subscale score. The interaction between the non-TKA group and time also remained significantly associated with a decreasing KOOS-QoL subscale score $(-0.15$ [0.07]; $P=0.0226)$ over the 4-year period.

Figure 2 shows the KOOS-QoL subscale mean scores over the 4-year period by TKA/OA status at baseline. Compared with the no knee OA group, the pre-TKA group had the worst QoL, followed by the non-TKA group and the post-TKA group. There was a significant interaction effect between time and QoL among the four groups (no knee OA, non-, pre-, and post-TKA), even after adjusting for the covariates $(\mathrm{F}=45.5, d f=19, P<0.0001)$. Moreover, although the post-TKA group showed better QoL over the 4-year follow-up period, this group also showed a decline in QoL scores after 2-year follow-up.

\section{Discussion}

The relationship between TKA/OA status at baseline and QoL over a 4-year follow-up period and were examined and compared to participants with no knee OA. The rate of decline in the KOOS-QoL score was 0.38 points per year over the 4-year period, after controlling for all covariates. We found that participants in the non-, pre-, and post-TKA 
Table I Baseline descriptive characteristics of the sample, $n=4,674$

\begin{tabular}{|c|c|c|c|c|c|}
\hline Variables & $\begin{array}{l}\text { No knee OA } \\
N=3,7 \mid \text { I (79\%) }\end{array}$ & $\begin{array}{l}\text { Non-TKA } \\
\mathrm{N}=902(19 \%)\end{array}$ & $\begin{array}{l}\text { Pre-TKA } \\
\mathrm{N}=36(1.5 \%)\end{array}$ & $\begin{array}{l}\text { Post-TKA } \\
\mathrm{N}=\mathbf{2 5}(0.5 \%)\end{array}$ & $P$-value \\
\hline Age in years & $61.2 \pm 9.1$ & $61.4 \pm 9.2$ & $60.0 \pm 9.7$ & $69.8 \pm 7.1$ & $<0.0001$ \\
\hline Gender & & & & & 0.0385 \\
\hline Male & $\mathrm{I}, 505(4 \mathrm{I})$ & $4 I I(46)$ & $17(47)$ & $12(48)$ & \\
\hline Female & $2,206(59)$ & $491(54)$ & $19(53)$ & $13(52)$ & \\
\hline Race & & & & & $<0.0001$ \\
\hline White or Caucasians & $2,959(80)$ & $676(75)$ & $18(50)$ & $24(96)$ & \\
\hline African American/Asian/non-whites & $752(20)$ & $226(25)$ & $18(50)$ & I (4) & \\
\hline Education & & & & & 0.0303 \\
\hline High school or less & $588(16)$ & $166(18)$ & II (3I) & $5(20)$ & \\
\hline More than high school & $3,123(84)$ & $736(82)$ & $25(69)$ & $20(80)$ & \\
\hline Marital status & & & & & 0.0115 \\
\hline Married & $2,444(66)$ & $305(34)$ & $20(56)$ & $4(16)$ & \\
\hline Unmarried/divorced/widow & I,267 (34) & $597(66)$ & $16(44)$ & $21(84)$ & \\
\hline Income per year in US dollars & & & & & $<0.000$ I \\
\hline$<50,000$ & $\mathrm{I}, 358(37)$ & $362(40)$ & $27(75)$ & II (44) & \\
\hline$>50,000$ & $2,353(63)$ & $540(60)$ & $9(25)$ & $14(56)$ & \\
\hline Health care coverage & & & & & 0.0189 \\
\hline Yes & $3,553(96)$ & $852(94)$ & $31(86)$ & $24(96)$ & \\
\hline No & I58 (4) & $50(6)$ & $5(14)$ & I (4) & \\
\hline Charlson comorbidity index & & & & & $<0.0001$ \\
\hline 0 & $2,800(84)$ & $621(79)$ & $16(59)$ & $16(84)$ & \\
\hline$>1$ & $538(16)$ & $167(2 \mid)$ & II (4I) & $3(16)$ & \\
\hline Depressive symptoms & & & & & $<0.0001$ \\
\hline CESD $<16$ & $3,366(91)$ & $786(87)$ & $24(67)$ & $22(88)$ & \\
\hline CESD $>16$ & $345(9)$ & $116(13)$ & $12(33)$ & $3(12)$ & \\
\hline $\mathrm{BMI}, \mathrm{kg} / \mathrm{m}^{2}$ & $28.4 \pm 4.8$ & $29.8 \pm 4.8$ & $31.5 \pm 5.9$ & $29.9 \pm 4.5$ & $<0.0001$ \\
\hline PASE & $160.7 \pm 82.4$ & $161.0 \pm 83.3$ & $134.9 \pm 89.5$ & $|2| . \mid \pm 70.4$ & $<0.0001$ \\
\hline KOOS-QoL & $69.7 \pm 28.3$ & $52.3 \pm 19.7$ & $39.0 \pm 22.9$ & $66.0 \pm 26.6$ & $<0.0001$ \\
\hline
\end{tabular}

Note: Values are presented as mean \pm SD or $n(\%)$.

Abbreviations: BMI, body mass index; CESD, Center for Epidemiologic Studies Depression Scale; KOOS-QoL, Knee Injury and Osteoarthritis Outcome Score-Quality of Life subscale; OA, osteoarthritis; PASE, Physical Activity Scale for Elderly; SD, standard deviation; TKA, total knee arthroplasty.

Table 2 General linear mixed model estimates for the KOOS-QoL subscale score over a 4-year period by TKA or knee OA status at baseline, $n=4,674$

\begin{tabular}{|c|c|c|c|c|}
\hline Predictor variables & $\begin{array}{l}\text { Model I } \\
\text { estimate (SE) }\end{array}$ & $P$-value & $\begin{array}{l}\text { Model } 2 \\
\text { estimate (SE) }\end{array}$ & $P$-value \\
\hline Intercept & $51.22(2.83)$ & $<0.0001$ & $61.74(2.95)$ & $<0.0001$ \\
\hline Time & $-0.38(0.03)$ & $<0.000$ I & $-0.38(0.03)$ & $<0.0001$ \\
\hline \multicolumn{5}{|l|}{ Main effect } \\
\hline No knee OA & Reference & & Reference & \\
\hline Non-TKA & $-14.28(0.74)$ & $<0.0001$ & $-13.97(0.73)$ & $<0.000$ I \\
\hline Pre-TKA & $-21.50(3.4 I)$ & $<0.000$ I & $-21.34(3.57)$ & $<0.0001$ \\
\hline Post-TKA & $-9.42(3.91)$ & 0.0161 & $-9.68(3.94)$ & 0.0143 \\
\hline \multicolumn{5}{|l|}{ Interaction with time } \\
\hline No knee OA $\times$ Time & Reference & & Reference & \\
\hline Non-TKA $\times$ Time & $-0.15(0.07)$ & 0.0302 & $-0.16(0.07)$ & 0.0226 \\
\hline Pre-TKA $\times$ Time & $-0.50(0.31)$ & 0.1107 & $-0.25(0.34)$ & 0.4561 \\
\hline Post-TKA $\times$ Time & $0.16(0.36)$ & 0.6626 & $0.15(0.37)$ & 0.6821 \\
\hline
\end{tabular}

Notes: Model I: Adjusted for time, age, sex, race, marital status, education, annual income, and health care coverage. Model 2: Adjusted for depressive symptoms, PASE, BMI, comorbidities along with variables in Model I.

Abbreviations: BMI, body mass index; KOOS-QoL, Knee Injury and Osteoarthritis Outcome Score-Quality of Life subscale; OA, osteoarthritis; PASE, Physical Activity Scale for Elderly; SE, standard error; TKA, total knee arthroplasty. 

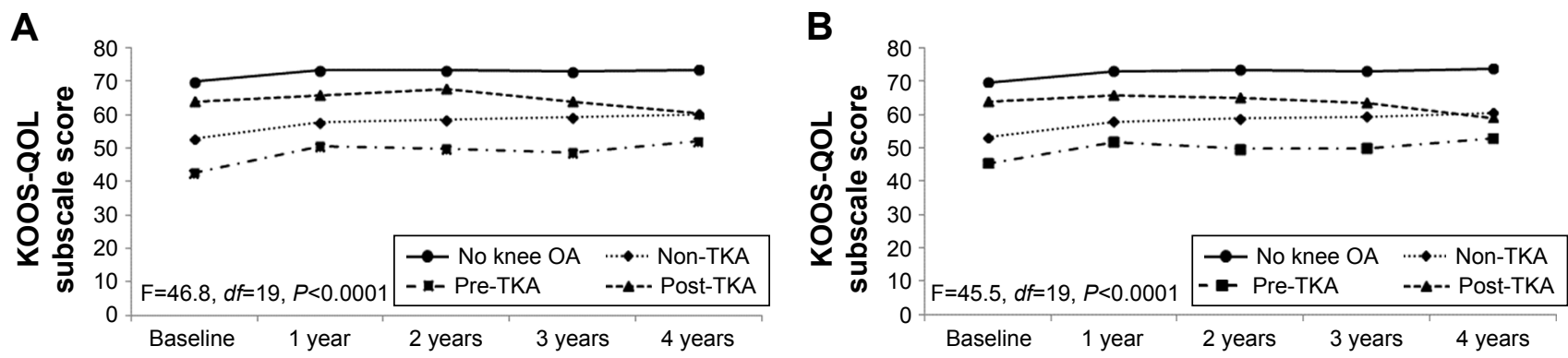

Figure 2 Means for the KOOS-QoL subscale score over a 4-year period, by TKA or knee OA status at baseline, $n=4,674$.

Notes: (A) Adjusted for sociodemographic variables. (B) Adjusted for sociodemographic and health variables.

Abbreviations: CG, ; KOOS-QoL, Knee Injury and Osteoarthritis Outcome Score-Quality of Life subscale; OA, osteoarthritis; TKA, total knee arthroplasty.

groups at baseline were associated with decreased QoL as compared with the no knee OA group, even after controlling for all covariates. The association was robust for the nonand pre-TKA groups. We also found an association between the non-TKA group and time, even after controlling for all covariates.

We found that the non- and pre-TKA groups were associated with greater decreases in the KOOS-QoL subscale scores. Interestingly, the KOOS-QoL subscale score in the non-TKA group significantly declined over the 4-year follow-up period. Comparatively, after adjusting for sociodemographic and health covariates, the post-TKA group had a lower decrease in the KOOS-QoL subscale score than the non- and pre-TKA groups. This confirmed the hypothesis that TKA would minimize the decline in patient-reported QoL over the 4-year follow-up period.

Participants in the post-TKA group were, on average, 9 years older than participants in the other groups, and had lower levels of physical activity. Intriguingly, these participants also showed a decline in the mean KOOS-QoL subscale scores after the 2-year follow-up period. The reasons for this decline in QoL after the 2-year mark are not well understood. Two recent papers found no association among age, physical activity, and KOOS-QoL scores. ${ }^{21,22}$

The current results are in line with other previously published studies. ${ }^{8,17,23,24}$ For example, KOOS was used a primary outcome measure in a single-center, randomized, double-blind study of 129 knees in 115 patients, where functional outcomes pre- and post-TKA (1- and 3-year follow-up) were measured. In that study, the average patient age was 70 years, and $67(58.3 \%)$ participants were female. The authors found a statistically significant association between the mean subscores (pain, symptoms, sport/recreation, and QoL) for the KOOS and TKA.

\section{Strengths and limitations}

The present study has several strengths. First, data from a multicenter, ongoing, prospective cohort study were used. Second, patient-reported QoL was examined using the KOOS-QoL subscale, which is a more contemporary outcome measure developed for more active patients. ${ }^{14}$ Third, patient-reported QoL over the 4-year follow-up period was examined in a well-defined community sample, who were grouped according to their TKA/OA status. Fourth, there were no dropouts, and data from the no knee OA group were used as a comparison. Finally, time-dependent effects were determined using a mixed models analysis.

However, the study has some limitations. First, the measure of QoL was self-reported, and QoL may have different meanings for different participants. Second, there was a lack of information regarding biomarkers, knee muscle strength, or knee flexion ranges of the participants, and no data pertaining to surgical procedure or techniques used to manage the condition during the study period. ${ }^{25}$ Third, most of the participants were Caucasian; thus, it may not be relevant to extrapolate the results of this study to describe other races. Finally, because the number of participants in TKA groups falls below the number of participants in the other groups, limited statistical power due to small sample size should be considered when interpreting the findings.

\section{Conclusion}

The present study is the first aimed at examining the relationship between TKA/OA status at baseline and QoL over a 4-year follow-up period, with the data compared to participants with no knee OA. The results of this study indicate that participants with a non-, pre-, or post-TKA status were all associated with a lower QoL. However, this association changed longitudinally in the non-TKA group. The slopes related to the data changed in all groups over the 4-year follow-up. Future studies are needed to understand the factors that influence QoL over time, particularly after TKA. 


\section{Acknowledgments}

The author extends his appreciation to the Research Center, College of Applied Medical Sciences, and the Deanship of Scientific Research at King Saud University for funding this research. The OAI is a public-private partnership comprised of five contracts (N01-AR-2-2258; N01-AR-2-2259; N01AR-2-2260; N01-AR-2-2261; N01-AR-2-2262) funded by the National Institutes of Health and Private funding partners. This manuscript was prepared using an OAI public use data set and does not necessarily reflect the opinions or views of the OAI investigators, the $\mathrm{NIH}$, or the private funding partners.

\section{Disclosure}

The author reports no conflicts of interest in this work.

\section{References}

1. McCalden RW, Hart GP, MacDonald SJ, Naudie DD, Howard JH, Bourne RB. Clinical results and survivorship of the GENESIS II total knee arthroplasty at a minimum of 15 Years. J Arthroplasty. 2017;32(7): 2161-2166.

2. Grayson CW, Decker RC. Total joint arthroplasty for persons with osteoarthritis. PM R. 2012;4(5 Suppl):S97-S103.

3. Patel A, Pavlou G, Mujica-Mota RE, Toms AD. The epidemiology of revision total knee and hip arthroplasty in England and Wales: a comparative analysis with projections for the United States. A study using the National Joint Registry dataset. Bone Joint J. 2015;97-B(8): 1076-1081.

4. Wylde V, Dieppe P, Hewlett S, Learmonth ID. Total knee replacement: is it really an effective procedure for all? Knee. 2007;14(6):417-423.

5. Bourne RB, Chesworth BM, Davis AM, Mahomed NN, Charron KD. Patient satisfaction after total knee arthroplasty: who is satisfied and who is not? Clin Orthop Relat Res. 2010;468(1):57-63.

6. da Silva RR, Santos AA, de Sampaio Carvalho Junior J, Matos MA. Quality of life after total knee arthroplasty: systematic review. Rev Bras Ortop. 2014;49(5):520-527.

7. Collins NJ, Roos EM. Patient-reported outcomes for total hip and knee arthroplasty: commonly used instruments and attributes of a "good" measure. Clin Geriatr Med. 2012;28(3):367-394.

8. Naili JE, Wretenberg P, Lindgren V, Iversen MD, Hedstrom M, Brostrom EW. Improved knee biomechanics among patients reporting a good outcome in knee-related quality of life one year after total knee arthroplasty. BMC Musculoskelet Disord. 2017;18(1):122.

9. Deshpande PR, Rajan S, Sudeepthi BL, Abdul Nazir CP. Patient-reported outcomes: a new era in clinical research. Perspect Clin Res. 2011;2(4): $137-144$.

10. van Cranenburgh OD, Prinsen CA, Sprangers MA, Spuls PI, de Korte J. Health-related quality-of-life assessment in dermatologic practice: relevance and application. Dermatol Clin. 2012;30(2):323-332, x.
11. Ko V, Naylor J, Harris I, Crosbie J, Yeo A, Mittal R. One-to-one therapy is not superior to group or home-based therapy after total knee arthroplasty: a randomized, superiority trial. J Bone Joint Surg Am. 2013; 95(21):1942-1949.

12. Moller E, Weidenhielm L, Werner S. Outcome and knee-related quality of life after anterior cruciate ligament reconstruction: a longterm follow-up. Knee Surg Sports Traumatol Arthrosc. 2009;17(7): 786-794.

13. Wylde V, Learmonth I, Potter A, Bettinson K, Lingard E. Patientreported outcomes after fixed- versus mobile-bearing total knee replacement: a multi-centre randomised controlled trial using the Kinemax total knee replacement. J Bone Joint Surg Br. 2008;90(9):1172-1179.

14. Roos EM, Toksvig-Larsen S. Knee injury and Osteoarthritis Outcome Score (KOOS) - validation and comparison to the WOMAC in total knee replacement. Health Qual Life Outcomes. 2003;1:17.

15. Peer MA, Lane J. The Knee Injury and Osteoarthritis Outcome Score (KOOS): a review of its psychometric properties in people undergoing total knee arthroplasty. J Orthop Sports Phys Ther. 2013; 43(1):20-28.

16. Roos EM, Lohmander LS. The Knee injury and Osteoarthritis Outcome Score (KOOS): from joint injury to osteoarthritis. Health Qual Life Outcomes. 2003;1:64.

17. Farrokhi S, Chen YF, Piva SR, Fitzgerald GK, Jeong JH, Kwoh CK. The influence of knee pain location on symptoms, functional status, and knee-related quality of life in older adults with chronic knee pain: data from the osteoarthritis initiative. Clin J Pain. 2016;32(6):463-470.

18. Zeger SL, Liang KY. Longitudinal data analysis for discrete and continuous outcomes. Biometrics. 1986;42(1):121-130.

19. Cnaan A, Laird NM, Slasor P. Using the general linear mixed model to analyse unbalanced repeated measures and longitudinal data. Stat Med. 1997;16(20):2349-2380.

20. Vock DM, Davidian M, Tsiatis AA, Muir AJ. Mixed model analysis of censored longitudinal data with flexible random-effects density. Biostat. 2012;13(1):61-73.

21. Kahn TL, Schwarzkopf R. Does total knee arthroplasty affect physical activity levels? Data from the osteoarthritis initiative. J Arthroplasty. 2015;30(9):1521-1525.

22. Kuijer PP, Kievit AJ, Pahlplatz TM, et al. Which patients do not return to work after total knee arthroplasty? Rheumatol Int. 2016;36(9):1249-1254.

23. Aunan E, Naess G, Clarke-Jenssen J, Sandvik L, Kibsgard TJ. Patellar resurfacing in total knee arthroplasty: functional outcome differs with different outcome scores: a randomized, double-blind study of 129 knees with 3 years of follow-up. Acta Orthop. 2016;87(2):158-164.

24. Mugnai R, Digennaro V, Ensini A, Leardini A, Catani F. Can TKA design affect the clinical outcome? Comparison between two guidedmotion systems. Knee Surg Sports Traumatol Arthrosc. 2014;22(3): 581-589.

25. Pua YH, Seah FJ, Clark RA, Lian-Li Poon C, Tan JW, Chong HC. Factors associated with gait speed recovery after total knee arthroplasty: a longitudinal study. Sem Arthritis Rheum. 2017;46(5):544-551.

26. The Osteoarthritis Initiative (OAI). San Francisco: The Coordinating Center, University of California San Francisco. Available from: http:// oai.epi-ucsf.org/datarelease/. Accessed March 8, 2018.
Patient Preference and Adherence

\section{Publish your work in this journal}

Patient Preference and Adherence is an international, peer-reviewed, open access journal that focuses on the growing importance of patient preference and adherence throughout the therapeutic continuum. Patient satisfaction, acceptability, quality of life, compliance, persistence and their role in developing new therapeutic modalities and compounds to optimize

\section{Dovepress}

clinical outcomes for existing disease states are major areas of interest for the journal. This journal has been accepted for indexing on PubMed Central. The manuscript management system is completely online and includes a very quick and fair peer-review system, which is all easy to use. Visit http://www dovepress.com/testimonials.php to read real quotes from published authors. 